\title{
A Multi-Agent System to Support ICT based Distance Learningthrough Modeling of Learner Needs: The Caseof Bachelor of Education at the University of Nairobi
}

\author{
YonahEtene $^{1}$, Patrick Owoche ${ }^{2}$,Dr.RobertOboko ${ }^{3}$ \\ ${ }^{I}$ Department of Computer Science, Kibabii University Kenya \\ ${ }^{2}$ Department of Information Technology, Kibabii University - Kenya \\ ${ }^{3}$ Department of Computer Science, University of Nairobi Kenya
}

\begin{abstract}
In distant learning environments, most learners need feedback from their lecturers and administration. The feedback supports them to achieve their learning objectives. However, the feedback that the most distant learners receive through the student's portal system is not adequate. This leads to learners using a lot of the scarceresources travelling to college to seek guidance on academic and administrative issues. This study proposes a multi-agent system that can provide personalized feedback to learners, lectures and administration in an efficient and effective way. The distant learners' need updates on fees, center meetings, examination results, assignments, study schedules etc. The feedback system provides feedback to the learner using the learners most appropriate mode, either sending an email alert, short message services or for clarification the learner can request for a phone call from the administration. The alerts provide a detailed summary of the information posted on the University website.
\end{abstract}

Keywords: Classifiers, Distant learners, Feedback, Learner needs, Multi-agent system

\section{Introduction}

An essential aspect of any distance education project is the support services that are available to the students [1]. Distance Learners cannot not easily achieve success in distance learning environment if they are not provided with learner support system. Understanding the learners is critical in providing appropriate support services for the survival of these learners [2]. Administrators and staff need to "explore strategies that will respond to the individual needs of learners' in a new learning environment [3],[4]. Most distance learners, mainly need feedback and encouragement, administrative procedures such as timely and accurate information, guidance and information, communication to both lecturer and other learners', and provision of accurate and clear procedures and access to help when they have problems [1].The characteristics of distance learners make them unique as compared to other learners in the traditional learning environment where learners get direct feedback on whatever they need.

Multi-agent approach applies very well to domains where distance, co-operation among different entities and integration of different modules of software, are of critical importance. Multi-agent systems (MAS) allow the modeling of learning as the emergent result of rich and coherent interactions, which occur over time, betweenhuman and artificial agents [5]. An agent can be a reactive or a more deliberative entity, able to communicate with other agents, solve problems based on a goal-oriented behavior, and act on behalf of a human user. The main advantage of using multi-agent technology is due to the flexibility resulting from the interactions agents may carry out.

Currently, there is inadequate of a feedback module in the students' management portal that learners can use to give real time feedback to the learners from the administration and lecturers basing on the learner's mode of feedback delivery. For filling this gap, we consider a MAS feedback model that uses intelligent agents to act on behalf of the administration and lecturers to deliver timely feedback [11] to learners by analyzing the learners' personalneed.

This paper is organized as follows: section two discusses some related works in intelligent agents based systems used in learning environment and learner support needs. Section three presents the proposed feedback system uses agents and Naïve Bayes classifier. Finally, section four describes respectively the results obtained and conclusions.

\section{Intelligent Agents Based Systems}

\section{Literature Review}

The interactions between agents can be either a direct agent-to-agent interaction or an indirect interaction. Indirect interactions are based on the environment. In the indirect interaction, an agent modifies another agent's environment triggering a reaction. An agent is a component of software or hardware, which are 
capable of acting exactingly in order to accomplish tasks on behalf of their users. MAS is an organization of coordinated autonomous agents which interact in order to achieve common goals. The major potential of multiagent systems relates to the ability of agent systems to support personalized and informal learning. The introduction of concepts such as MAS allows web technology to improve the process of modeling and designing for distance learning [7]. The ubiquitous learning environment provides an interoperable, pervasive, and seamless learning architecture to connect, integrate, and share three key dimensions of learning resources. These are learning collaborators, learning contents, and learning services [7]. The learner voice is a crucial aspect in promoting learning and so it requires listening to learners' opinions and involving them in planning. When considered it can help one to recognize and accommodate learners' individual needs.

\section{Learner Support Needs}

In day-today life, people seek specific knowledge and skills or services, when and where they need it. ICT makes information and knowledge available in real time and provides opportunities for continuing and lifelong learning. It is driven by each learner's personal needs and the feedback can be customized.

According to Lewis and Spencer [8], the key roles of manager/teacher should be identified and included in the open learning schemes when supporting distance learners. These involve services such as informing, advising, guiding, tutoring and counseling. These services may be communication, facilitation, feedback, reassuring, listening, inspiring, valuing and encouraging. Distance learners need feedback services more to support them while off campus. They can make enquiries from administration/lecturers and receive feedback in real time using the most appropriate mode of delivery to the learner. This approach will help solve problems related to delayed feedback delivery such as discouragement, increased costs and time consumed whenever the learner travels to seek for the required information from the administration and lecturers.

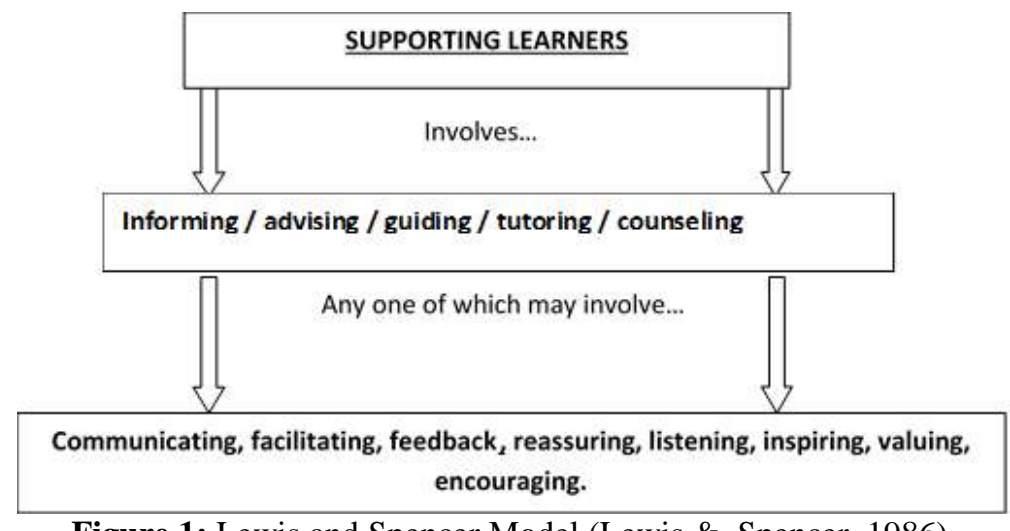

Figure 1; Lewis and Spencer Model (Lewis \&. Spencer, 1986)

\section{Classifiers}

Naïve Bayes classification is based on Bayes theorem. A simple Bayes classification namely the Naïve classifier is comparable in performance with decision tree and neural network classifiers. Naïve Bayes classifiers have also exhibited high accuracy and speed when applied to large database. Naïve Bayes classifier assumes that the effect of an attribute value on a given class is independent of the values of the other attributes. This assumption is called class conditional independence. It is made to simplify the computations involved and, in this sense, is considered "naïve". While applying Naïve Bayes classifier to classify text, each word position in a document is defined as an attribute and the value of that attribute to be the word found in that position. Naïve Bayes is formalized as the product of the prior probability which is based on previous experience and the likelihood of a given attribute being in a given class, this forms the posterior probability. To classify an unlabeled example, it is just a matter of using the prior probabilities of a given category and multiplying them together. The category which produced the highest probability would be the label/classification for the unlabeled example. Only the words found in the unlabeled example would be looked up in the feature vector.

Equation (i)would be used to classify an unlabeled example. Given a document $d$ and a class c, where the goal is to predict the probability that the document $\mathrm{d}$ belongs to class $\mathrm{c}$.

$\mathrm{P}(\mathrm{c} / \mathrm{d})=\operatorname{argmax}(\mathrm{P}(\mathrm{d} / \mathrm{c}) \cdot \mathrm{P}(\mathrm{c})))$

\section{System Architecture}

This section highlights the general architecture of the feedback system and the agents that are involved in the feedback system and their functionalities as in figure 2.Based on the MAS approach, we designed a MAS system whose purpose is, to support ICT based distant learning through modeling of learner needs. The system 
provides feedback to learners by sending email alerts, short messages to learners basing on his/her need. The system also gives option for the learner to also request for a phone call, which is only made by the administrator.A counter and billing system has been built into the system to enable management to bill the learner who requests for clarification of information through phone call.

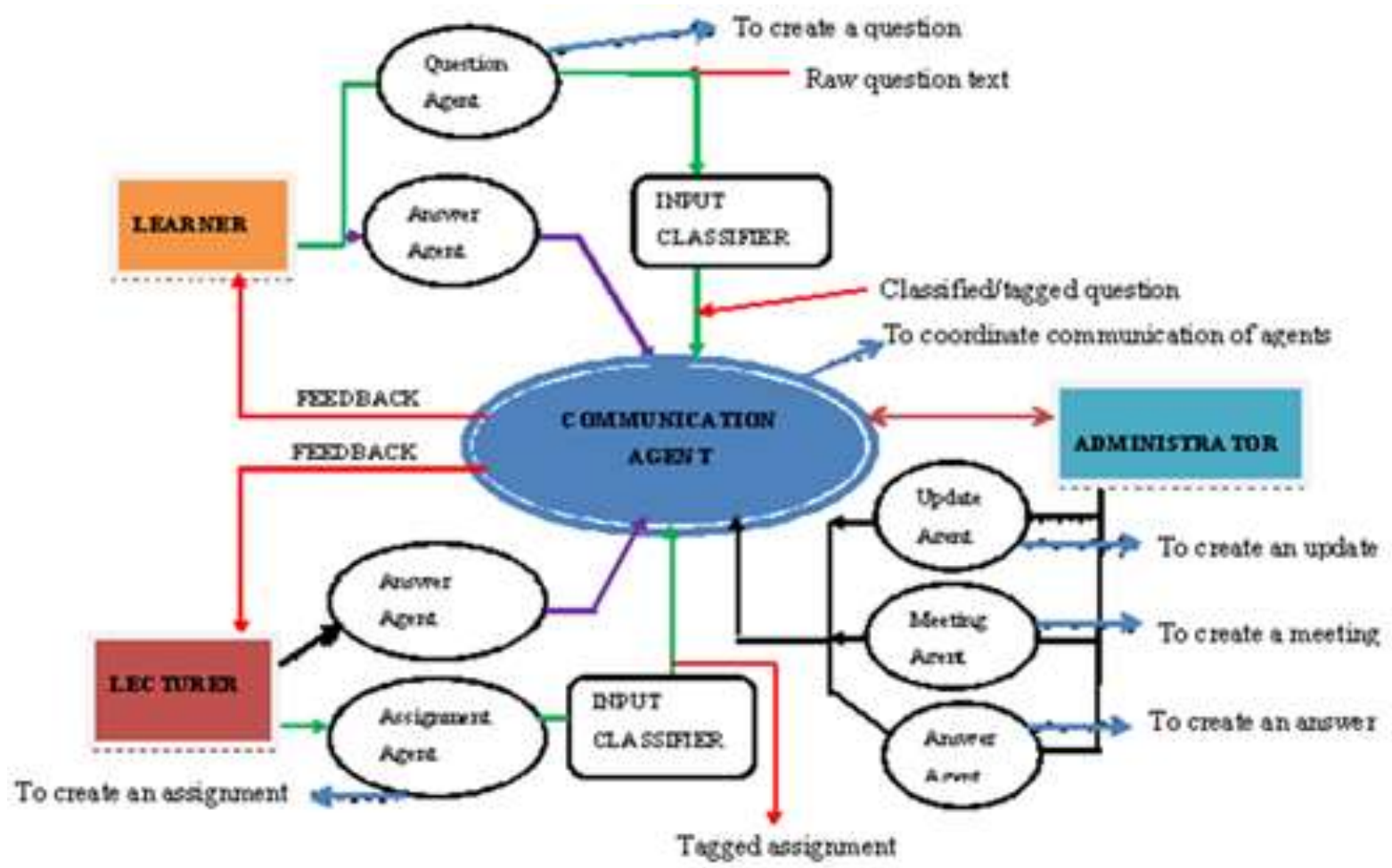

\section{Two waycommunication}

Fig 2 General structure of the proposed system

\section{General Operation}

The communications agent is also known as the coordinator agent. It coordinates all communication in the system. All other agents forward information to this agent for routing to the desired destination.

\section{Coordinator}

It's the master agent in the system that coordinates all the other agents in the system. It coordinates communication of information to the different stakeholders in the system i.e. students, lecturers and administration. It reads the labels on the message send to it by another agent, interprets it and routes to the right destination. This agent sends out alerts (feedback) in the form of emails, short message to the learner or places a phone call requests. The default alert is email. The other two may be used only upon request by the learner. Phone call requests can only be used for clarification of information and only the administrator is allowed to make a phone call to the learner and subsequently bill him/her through the system.

\section{Question agent}

This agent is used by students to create and post questions to the lecturers or fellow students. It then classifies the question using the classifier- Naïve Bayes classifier Model basing on natural language processing (NLP). It then passes the question to the communications agent.

\section{Answer agent}

It gets the answer created, wraps it over into an answer object and then sends it over to the person who asked the question.

\section{Assignment agent}

The lecturer creates an assignment and then the assignment agent takes the assignment wraps it up into an assignment object with a tag and forwards to the communication agent to forward to the intended recipient. 
Update agent

It receives the Updates from the user agent, it wraps it up into updates object and then sends it over to the Communication Agent.

\section{Meeting agent}

It creates a meeting and wraps it up into a meeting object and with a tag; who it is intended for and the sends it over to the Communication agent to route to the intended persons. Therefore, these agents cooperate with each other to help and assist learners in the learning process, they send requests to the administrators and lecturers and take the feedback from the administrators and lecturers back to the learners.

\section{System Design and Development}

\section{Methodology}

This section highlights the steps followed in the design of the system and the initial study that informed the development of the proposed systems to support distance learners.By examining the general domain of ICT- learner support services, this paper seeks to meet the needs of the distance learners' by providing feedback as informed by an initial research on learner needs. The characteristics of distance learners make them unique as compared to other learners in the traditional learning environment where learners get direct feedback on whatever they need.

Distance learners need clear, complete and timely information about:

i. Availability of academic services such as counseling, advising, feedback on assignments etc.

ii. Performance expectations concerning datelines, study time requirements and attendance.

iii. When, where and how learner/instructor interaction takes place (face-to-face) meetings, residential events, telephone etc.

The initial study was conducted in order to gain some background data on the intended responses and also to gain more information from the distant learners on whether they were getting feedback from the University, the mode of feedback delivery used, to support their learning and the challenges they were facing. From the analysis of 20 samples, it was found that distant learners needed feedback. However, the needs were different from one learner to another, hence the need to design a system that can analyze, classify and meet individual learner needs by providing timely feedback to support the learning process.

The system was developed using Agile development methodology. This methodology was preferred because it is iterative and incremental and so allows the assessment of the projects direction throughout the development lifecycle.

\section{System Implementation}

\section{Results}

The system was implemented using multi-agent technology. The programming language used waspython.It was suitable because it can implement multi agents and most of its features are already in built within the language making it more cost effective.

\section{System Evaluation}

After developing the system and implementing it, it was evaluated by the learners to see whether it met the development objective, i.e. a model to support ICT based distance learners, while off campus by providing timely feedback to the learners. The final model was tested with the bachelor of education distance learners of the University of Nairobi. The outcome was to provide information whether it will meet the distance learner needs in a distance learning environment [9]. The results of an assessment process provided information used to determine whether or not intended objectives were achieved. The university has distant learners in different regions in Kenya. The feedback system was hosted by python anywhere so that it could be accessed by the learners easily.

The learners used the system for a period of two weeks. At the end of the two weeks, they were required to evaluate the system based on usability and functionality.ata was collected by interview and a structured questionnaire. The questions were based on an inventory of questions developed by the Presences research working group at the Technische University Eindhoven, Netherlands. The questionnaire had two sections; the first section had a set of questions related to usability and second section on functionality.

A 5 - point Likert type scale was adopted for the second section of the questionnaire while the first section of the questionnaire respondents were required to respond with "Yes or No".

The questionnaire was used because of two reasons:

(a) It is an effective small-scale research tool, and

(b) Because "the knowledge needed is controlled by the questions, therefore it affords a good deal of precision and clarity" [10]. 
Usability

\section{Discussion}

From the results obtained on the usability and tabulated as shown in table 1. Twenty one (21) learners had accounts on the feedback system and were able to receive fees updates, meeting notices through the system.Twenty one (21) distant learners felt that the feedback received was timely and was received through their preferred individual mode.Nineteen (19) learners felt that the system was good as they were able to receive timely feedback through it and also through the desired mode.

\begin{tabular}{|l|l|l|l|}
\hline No. & Question & Yes & No \\
\hline 1. & Are you getting feedback from the system? & 21 & 0 \\
\hline 2. & Was the feedback timely? & 19 & 2 \\
\hline 3. & Did you get updates about fees, meetings from the administration? & 21 & 0 \\
\hline 4. & Did you receive the feedback through the desired mode? & 21 & 0 \\
\hline 5. & Do you have an account on the feedback system? & 21 & 0 \\
\hline
\end{tabular}

\section{Functionality}

From the results obtained on the functionality of the system and tabulated as shown in Table 2 . Nineteen (19) of the distant learners agreed that the site was easy to navigate, while 2 learners disagreed. The learners who disagreed with the functionality of the system citedthe reason of having poor computer skills necessary to access and navigate through thesystem.Eighteen (18) learners agreed that the information on the system was clear and well organized. However, three learners disagreed as they had not interacted with the system in totality.Twenty (20) distant learners agreed that the system was good enough to use to support them and would recommend the same to others. However, one (1) learner was neutral while the other two (2) disagreed with it.

\begin{tabular}{|l|l|l|l|}
\hline No. & Question & Yes & No \\
\hline 1. & Are you getting feedback from the system? & 21 & 0 \\
\hline 2. & Was the feedback timely? & 19 & 2 \\
\hline 3. & Did you get updates about fees, meetings from the administration? & 21 & 0 \\
\hline 4. & Did you receive the feedback through the desired mode? & 21 & 0 \\
\hline 5. & Do you have an account on the feedback system? & 21 & 0 \\
\hline
\end{tabular}

From the system users it was evident from the results that the system is easy to navigate, had well organized information and can support the distant learners while off campus. They were able to get through the navigate through it, place individual requests to the administration, staff and receive their feedback in a timely manner. They were also updated on any new developments in the University in a timely manner through their individual preferred mode as selected on first logon to the system.

\section{Conclusion}

This paper proposed a multi-agent feedback system which can analyze learner's needs and send feedback to the individual learner using their preferred mode. Also, one of the agents of the system can coordinate the information communication to learners and university staff, just like a human being and then route the information to the right person and with the right mode as requested by the learner.

The prototype addressed the following limitations of the feedback the learners were getting from the University.Real time notification to the learners, to ensure that learners don't missonimportant updates and information posted on theuniversitywebsite. Mode of feedback delivery: learners are able to receive timely feedback from academic and administrative units using the mode they mostly prefer as individuals; hence addressingtheindividual needs of feedback delivery and by so reducingon time wastage and cost since learners can get information in real time.

\section{Acknowledgements}

We acknowledge the School of Computing and Informatics, University of Nairobi, for granting us unlimited access to computing resources.

\section{References}

[1]. P. Muchiri (2012), Construction and evaluation of the effectiveness of a needs-based learner support system: the case of the school of continuing and distant education, University of Nairobi-Kenya GRL LC 5800 M\&3

[2]. Ji-Yeon, (2000), The current status of learner support in distance education. Emerging issues and future research agenda.

[3]. Van Dusen, (1997), The virtual campus: Technology and reform in higher education. ASHE-ERIC Higher Education Report Vol. 25 No.5 Washington, DC: US Department of Education. 
[4]. P. Saelens, (2004). Defining best practices for distance learners provided by student support centres. Arizona State University.

[5]. C. Webber, S.,Pesty, N.,\&Balacheff, (2002). "A multi-agent and emergent approach to learner modeling", Proceedings of the15th European Conference on Artificial Intelligence (ECAI2002), F. Van Harmelen (ed.), IOS Press: Amsterdam, $2002, p p .98-102$.

[6]. A. Bennane (2010). Tutoring and multi-agent systems: Modeling from experiences.

[7]. Sung, J.-S. (2009). Application protocol design for collaborative learning. International Journal of u- and e- Service, Science and Technology, 2(4).

[8]. R. Lewis \&D. Spencer(1986), What is Open Learning? Cambridge: Cambridge University Press.

[9]. D. Nicol (2007), Principles of good assessment and feedback: Theory and practice. Proceedings of the REAP International Online Conference on Assessment Design for Learner Responsibility, 29-31 May, 2007. Retrieved from http://www.reap.ac.uk/reap/public/papers/Principles_of_good_assessment_and_feedback.pdf

[10]. M. Donough (1997), Precision and clarity of information.

[11]. D. Boud\& Associates (2010), Assessment 2020: Seven propositions for assessment reform in higher education. Sydney: Australian Learning and Teaching Council. Retrieved from http://www.iml.uts.edu.au/assessment-futures/Assessment2020_propositions_final.pdf 2 